\title{
Die Note des Barons von Serra vom 8. Mai 1813 an den sächsischen König Betrachtungen zu einem kaum bekannten Dokument der sächsischen Geschichte
}

\author{
von \\ RUDOLF JENAK
}

Unter den umfangreichen Beständen des Hauptstaatsarchivs Dresden, die Dokumente der Rheinbundära des Königreichs Sachsen beinhalten, stellen sowohl der Briefwechsel zwischen dem Kaiser Napoleon und dem König Friedrich August I. als auch die Archivalien, die mit der Konvention vom 20. April 1813, ${ }^{1}$ die Sachsen mit Österreich aushandelte und abschloss, ein besonders brisantes Material dar. Gemeint ist hiermit, dass eine wissenschaftliche Bearbeitung und anschließende Publizierung sehr lange unterblieb, da die Situation des Königreichs Sachsen nach dem Wiener Kongress es als äußerst unzweckmäßig erscheinen ließ, Interna und Details der Beziehungen zwischen dem Napoleonischen Frankreich und dem Königreich Sachsen in aller Öffentlichkeit auszubreiten.

$\mathrm{Zu}$ diesen bislang nicht oder nur sehr spärlich veröffentlichten Papieren zählt auch das Schreiben des damaligen französischen Gesandten, Baron von Serra, ${ }^{2}$ das dieser im

1 Rudolf Jenak, Die Realität der Österreichisch-sächsischen Konvention vom 20. April 1813, in: Mitteilungen des Vereins für sächsische Landesgeschichte, Neue Folge 5 (2007), S. 5-24.

2 Biografische Hinweise zur Person des Charles François de Serra: Der Baron von Serra wurde 1760 in einer alteingesessenen genuesischen Familie als Gian Carlo Serra geboren und trat bereits 1780 in den Dienst der Republik Genua. In dieser Eigenschaft erfüllte er politische Aufträge in Madrid und Paris. Ende der achtziger Jahre wurde er Mitglied des Kleinen Rates, der Regierung dieser Stadtrepublik. Zeitweilig war er als Diplomat der Republik Genua am Wiener Hof tätig. In welcher Hinsicht dieser Mann eine Rolle in antioligarchischen Verschwörungen innerhalb der Republik Genau spielte, erschließt sich aus den Hinweisen der unten genannten Italienischen Enzyklopädie nicht. Unter dem Einfluss der Französischen Revolution und der Politik des Direktoriums entstand um 1796 die Ligurische Republik, in deren Auftrag Serra in Paris tätig wurde. Nach der Vereinigung dieses Gebietes Norditaliens mit Frankreich trat er in den diplomatischen Dienst Frankreichs und wurde im Oktober 1807 zum Mitarbeiter der diplomatischen Residenz Frankreichs in Warschau. Sein besonderes Aufgabengebiet war die Wahrnehmung der Interessen des Kaiserlichen Frankreichs in Danzig. Dieser Dienst brachte ihn auch mit den Angelegenheiten des Königs von Sachsen, Herzog von Warschau, in nähere Berührung. In den Jahren 1810 und 1811 wurde der Baron von Serra, der auch Offizier der Ehrenlegion und Träger anderer hoher Auszeichnungen des Kaiserreichs Frankreich war, zum außerordentlichen und bevollmächtigten Gesandten Frankreichs am Hofe des Königs von Württemberg. Im Mai des Jahres 1811 wurde er von dort im gleichen Rang an den Hof des sächsischen Königs versetzt. Ende Oktober 1813, nach der Niederlage des Kaisers Napoleon bei Leipzig, verstarb der Baron von Serra in Dresden. Vgl. JacQues HenRI-RoberT, Dictionnaire des Diplomates de Napoleon, Paris 1990; Lessico Universale Italiano. Enciclopedia Italiano, Roma 1978: Serra, Gian Carlo (1760-1813) Fautore di una conspiratione antioligarchita, 
Auftrag Napoleons und auf der Grundlage von dessen Vorgaben ${ }^{3}$ erarbeitet und am 8. Mai 1813 Friedrich August I. vorgetragen hatte. Es ist in einer Abschrift des Originals innerhalb eines Bestandes der Korrespondenz des Ministers Senfft von Pilsach mit dem sächsischen Gesandten in Wien, dem General von Watzdorf, enthalten. ${ }^{4}$ Dieser höchst merkwürdige Vorgang der Übergabe des Schreibens an den sächsischen König ereignete sich in Prag, ${ }^{5}$ kurz nach dem Eintreffen der Nachricht vom Sieg der französischen Armee über die Truppen der Verbündeten am 2. Mai 1813 nahe der Stadt Lützen. Es ist offensichtlich, dass der Inhalt dieses Dokuments voraussetzte, über eine Bilanz der langjährigen, sehr vertrauten Beziehungen zwischen den beiden Monarchen zu verfügen, wie sie nur dem Kaiser geläufig sein konnte, nicht aber seinem Gesandten am Dresdner Hof. Insofern scheint es zweckmäßig zu sein, dieses Dokument eher als eine Deklaration des Kaisers Napoleon an den sächsischen König denn als einen von dem Baron von Serra verfassten Text zu werten. Für die Einordnung des Zwecks dieses Papiers in die Dokumente jener Epoche empfiehlt es sich, vielmehr von einer ,Note‘ als von einem ,Ultimatum' Napoleons an den sächsischen König zu sprechen.

Der Baron von Serra, der seit Ende Februar 1813 zusammen mit König Friedrich August I. und dessen Mitarbeiterstab den Weg über die Stationen Plauen, Regensburg bis Prag mitgereist war und die zweite Verbindung des Kaisers zum sächsischen Hof darstellte, bat am 7. Mai 1813 um eine Audienz beim König. Dieser empfing ihn, und von Serra teilte ihm das vom Kaiser persönlich bestätigte Ergebnis der Schlacht bei Lützen mit. ${ }^{6}$ Bei dieser Gelegenheit übergab der Baron von Serra dem König das bis dahin zurückgehaltene Schreiben des Kaisers Napoleon vom 14. April 1813. ${ }^{7}$ Während einer weiteren Audienz des Barons beim König Friedrich August I. am 8. Mai 1813 trug er ihm einen schriftlichen Text vor, dessen Kerngedanke darin bestand, herauszufinden, ob Friedrich August I. sich immer noch als Angehöriger des Rheinbundes ansehe, und ob er gewillt sei, die daraus erwachsenden Verpflichtungen zu erfüllen.

Der König beantwortete bei jener zweiten Audienz diese ihm schriftlich vorgelegte Frage des Kaisers ohne Umschweife und sehr direkt positiv. Ein daraufhin formulierter Brief an den Kaiser, datiert vom 10. Mai 1813, stellt die schriftliche Antwort auf die vom Baron von Serra übermittelte Frage dar. Die besagte Fragestellung erscheint als Annex zu diesem Brief. 8

ambasciatore a Parigi e Madrid per Repubblica, e poi per Napoleone in Polonia, autore di commentari storici; e, più importante di tutti, Gerolani, (v.) nomo politico et storico.

3 Das damit gemeinte Schreiben des Kaisers vom 20. April 1813 an den Baron von Serra, das zu den unveröffentlichten Briefen Napoleons zählt, wird im Anhang dieses Textes als 3. Titel in der Originalfassung vorgestellt.

4 Sächsisches Staatsarchiv - Hauptstaatsarchiv Dresden (im Folgenden: HStA Dresden), 10026 Geheimes Kabinett, Loc. 742/4, fol. 151v-152r.

5 Auf Einladung des österreichischen Kaisers Franz I. reiste König Friedrich August I. am 19. April 1813 von Regensburg über Linz nach Prag, um, wie er offiziell verlautbarte, seinen Völkern näher zu sein.

6 Über das Ergebnis der Schlacht gab es einige Tage lang Irritationen: Die unterlegenen Russen und Preußen meldeten nach allen Seiten ihren Sieg, wenngleich das Gegenteil zutraf. Diese Nachricht wirkte auf den König, den Minister Senfft und hohe sächsische Militärs tagelang äußerst verwirrend.

7 Das Original dieses Briefes trägt einen Zusatz von anderer Hand: Remise an Roi le 7. Mai 1813. par le Bn. de Serra dans une audience particulaire. Vgl. HStA Dresden, 10026 Geheimes Kabinett, Loc. 2760/15, fol. 30v.

8 Rudolf Jenak (Hg.), „Mein Herr Bruder ...“. Napoleon und Friedrich August I. Der Briefwechsel des Kaisers der Franzosen mit dem König von Sachsen 1806-1813, Beucha/ Markkleeberg 2010, Brief Nr. 151 vom 10. Mai 1813, Annex S. 158. 
Der bisher nicht veröffentlichte Originaltext der Note des Barons von Serra liegt in französischer Sprache vor. Er ist ein äußerst bedeutsames Dokument über die von Kaiser Napoleon als offensichtliche Treulosigkeit empfundenen Verhaltensweisen des Königs von Sachsen in den Tagen von Anfang April bis zum 7. Mai 1813. Dem Kaiser standen für die Erklärung dieser das Bündnis schädigenden Verhaltensweisen nur Vermutungen und Gerüchte sowie eine Reihe an ihn gerichteter und außergewöhnlich ausweichender Briefe des sächsischen Königs zur Verfügung. Beweise für begangenes Hintergehen der Bündnisverpflichtungen des Wettiners aber konnten ihm im Detail nicht bekannt sein.

Der Dreh- und Angelpunkt der beim französischen Kaiser auftretenden Zweifel an der Bündnistreue seines Herrn Bruder und Verbündeten, wie er den König von Sachsen in seinen Briefen freundschaftlich und achtungsvoll benannte, war die wiederholte Ablehnung der Forderung Napoleons, ihm für seine Offensive gegen die bis zur mittleren Saale vorgerückten verbündeten Preußen und Russen die sächsische Reiterei zur Verfügung zu stellen. Letztere war eine schlagkräftige und bewährte Offensivformation der sächsischen Armee, auf deren Einsatz Napoleon nicht verzichten wollte. Deshalb äußerte der Kaiser wiederholt den Wunsch, die zwei Kürassierregimenter in Stärke von 1.800 Mann bei Beginn der Offensive gegen die Verbündeten zur Disposition zu haben. Dieses Anliegen, dem König von hochrangigen französischen Stabsoffizieren wenigstens dreimal in kurzen Abständen überbracht, wurde von Friedrich August hartnäckig abgewiesen.

Die Ablehnungen seitens des sächsischen Königs - die immer wieder damit begründet wurden, dass er dem Kaiser diese Reiterei in ihrem momentan schlechten Zustand keineswegs anbieten könne, weil ihm dies zu peinlich wäre - waren für den scharfsinnigen Militär Napoleon, gerade angesichts der zugespitzten Situation kurz vor einer von ihm vorbereiteten Offensive gegen die Truppen der Verbündeten, allzu durchsichtig. Nach der dritten Ablehnung schrieb Napoleon, schon aufgebracht, doch immer noch höflich, dass er nicht begreifen könne, wieso der König den einzigen Beitrag, der ihm zur Befreiung seines Staates von den Truppen der Verbündeten abverlangt werde, verweigere.

Es war absolut folgerichtig, dass die Deklaration, die der Baron von Serra dem König vortrug, damit beginnt, auf die verweigerten beiden sächsischen Reiterregimenter Bezug zu nehmen. Der Baron von Serra formulierte seinen ersten Satz wie folgt: S. M. der Kaiser und König hat darum gebeten, seriöse Begründungen für die Motive der Weigerung des Königs, seine Reiterei zur Verfügung zu stellen, zu erhalten, obwohl S. M. der König von Sachsen ihm Unterstützung für seine Dispositionen in Aussicht gestellt habe. Zwei Regimenter Kavallerie und eine große Verbindlichkeit habe er in der Antwort an den General Flabaut bekundet. Nun aber habe er statt einer Antwort den Baron von Serra an den Grafen von Senfft verwiesen.

Der Kaiser, so lautet der Text, verstehe den Unterschied nicht, der sich gegenwärtig zu dem ruhigen und freundschaftlichen Verhalten des Königs seit sechs Jahren offenbare, wo Friedrich August eher ein Freund denn ein Alliierter gewesen sei. Falls er persönliche Wünsche des Königs nicht immer erfüllt habe, werde er dies berücksichtigen. Aber niemals werde er seinen Verbündeten im Stich lassen, und er erwarte daher auch vom König, dass er ebenso wie der Kaiser an der Spitze seiner Armee für die Befreiung Sachsens kämpfe und seine Bündnisaufgaben erfülle.

Der folgende Gedanke dieser Erklärung geht der Frage nach, ob der Seitenwechsel, den man aus den wiederholten Ablehnungen der Waffenhilfe ableiten kann, dem König Vorteile bringen könnte? Die Vermutung des Kaisers, der König verleugne inzwischen sein Herz, wenn er damit rechne, dass nun für ihn sehr vorteilhafte Beziehungen anderenorts vorhanden seien: Er, der Kaiser, habe in Jena, in Posen und 
Tilsit dem König die Hand gereicht. Von den zwei neuen Staaten in Europa habe der König einen, das Herzogtum Warschau, erhalten. Dessen Existenz wurde bewahrt und verteidigt.

Warum entfernt sich der König, der weise und treu sei, von diesen seinen Tugenden? Wohin ist das Vertrauen zu ihm geraten? Was ist es, das seine Güte, seine große Verbundenheit zu ihm, sein Vertrauen zunichte gemacht hat? Haben etwa die gemeinsamen Feinde verführerische Vorschläge gemacht? Oder hat sich in den Truppen ein böser, aufrührerischer Geist herausgebildet? Der Kaiser verweist darauf, dass der König im letzteren Fall über genügend Mittel verfüge, gegen Kommandeure mit derartigen Verhaltensweisen vorzugehen.

Napoleon, der mehrere Möglichkeiten für die erkennbare Untreue des Königs aufzählt, der natürlich auch über interne Informationen aus Wien, Regensburg und möglicherweise auch Prag verfügte, nennt sodann einen Verdacht, der den tatsächlichen Bedingungen sehr nahe kam: Der König möchte das Kavallerie-Korps für eine große Gelegenheit aufsparen, falls eine Balance eintrete, um rasch militärische Kräfte bereitstellen zu können. Der Kaiser meint mit Balance ganz offensichtlich ein denkbares Patt zwischen Frankreich und den Verbündeten, und er lässt offen, wem der sächsische König in einem solchen Fall mit seiner aufgesparten Kavallerie zu Hilfe käme. Dennoch ist seine Vermutung sehr treffend und seine Verdächtigung so formuliert, dass er daraufhin auf die Verantwortung des sächsischen Königs verweisen kann, durch sein Verhalten einen eigenen Beitrag zur Befreiung Sachsens durch Frankreich und den Rheinbund zu leisten, statt die Erreichung dieses Ziels zu verzögern.

In gleicher Weise kommt im Text dieses Dokuments des Barons von Serra ein weiterer wichtiger Beweggrund für die vermutete Untreue des sächsischen Königs zur Sprache: Der König habe stets sein großes Vertrauen zum Kaiser Napoleon betont. Ist nun, wo der Kaiser und König von einer Misere betroffen ist, da ihm die Launen der Natur ${ }^{9}$ in Russland so schädlich waren, ein Grund für Veränderungen in diesem Verhältnis?

In der Tat: Der Verlust des Glaubens an die Fortune Napoleons löste beim sächsischen König erhebliche Befürchtungen aus, dass dieser nicht in der Lage sein könnte, das Vordringen der Verbündeten nach Westen zu stoppen, und daher die Besitzungen in Polen in Gestalt des Herzogtums Warschau ihm unwiederbringlich verloren gehen würden. Daher suchte Friedrich August dringend nach einer Macht, die ihm im äußersten Falle gewisse Garantien für den Erhalt seiner Besitzungen in Deutschland und sogar Kompensationen für die bereits eingetretenen Verluste in Polen verschaffen würde. Er fand diese Macht in Gestalt des österreichischen Kaiserreiches und nutzte das Angebot des Kaisers Franz I., an der Mediation armé teilzunehmen, als scheinbar unverfänglichen Schirm, um hinter dem Rücken des französischen Kaisers einen umfassenden Allianzvertrag mit Österreich zu schließen.

Napoleon greift daher in dem vorliegenden Dokument die Argumentation des Königs ihm gegenüber auf, er wolle Österreich bei dessen Initiative unterstützen, zwischen den sich gegenüberstehenden Mächtegruppen zu vermitteln, um Europa einen dauerhaften Frieden zu verschaffen. Die Durchsichtigkeit und auch die Naivität dieser Erklärung des sächsischen Königs erleichterte es dem Kaiser Napoleon, klarzustellen: Diese Wünsche nach Frieden sind so, wie dieser Frieden selbst ein Gegenstand der Rechtfertigung ist; sie können den erzwungenen Wettlauf einer der interessierten Seiten, zu überleben, mit dem Gedanken, den Krieg zu gewinnen, nur durch die wirksamste Macht erreichen.

9 Vgl. Brief des Kaisers vom 18. Januar 1813: JenAK, Mein Herr Bruder (wie Anm. 8), Brief Nr. 130, S. 128-133. 
Damit legte Napoleon den Kern des Arguments, das der sächsische König als Motiv seines scheinbar harmlosen Anschlusses an das Habsburgerreich vorgebracht hatte, schonungslos und zutreffend bloß: Das Hintergehen des Bündnispartners aus dem Wunsch heraus, sich dem vermeintlich Stärkeren anzuschließen, und hierfür Zugeständnisse zu machen, die für einen Rheinbund-Partner unzulässig und schädlich waren. Allerdings, die vom Kaiser unterstellte und vermutete Furcht des Königs vor den militärischen Ereignissen spielte für Friedrich August I. in diesem Zusammenhang sicherlich keine besondere Rolle. Selbst das Argument des Königs, er baue seine Kavallerie erst wieder auf und könne daher unmöglich dem Kaiser eine derart unvollkommene Truppe anbieten, wird mit dem Gedanken, er solle zum militärischen Erfolg der Kampagne den Beitrag bereitstellen, den er leisten könne, ad absurdum geführt. Denn im Rahmen der Convention vom 20. April 1813 unterstellte Friedrich August I. dem österreichischen Oberkommando ca. 18.000 sächsische und polnische Soldaten sowie zwei bedeutende Festungen, nämlich Torgau und Königstein, die er damit zugleich seinem Rheinbund-Kontingent entzog. Das war die vom Kaiser unterstellte Rückversicherung des sächsischen Herrschers, falls es zu der besagten Balance der Kräfte kommen würde.

Am Ende dieses Papiers werden zwei Feststellungen hervorgehobenen: Friedrich August solle unverzüglich die beiden Kürassierregimenter bereitstellen und dorthin in Marsch setzen, wo sie benötigt werden. Nur das wäre bündnistreu und ein Dienst, der für Sachsen erfüllbar ist. Der Kaiser lege gerade auf einen solchen Akt der Liebenswürdigkeit des Königs als seines Verbündeten und Freundes größten Wert und würde es ihm für immer hoch anrechnen. Ferner solle der König an den Kommandanten der Festung Torgau den Befehl erteilen, dem General Reynier das Kommando über die Festung zu übergeben, und damit das dort versammelte sächsische Rheinbund-Kontingent dem 7. Armee-Korps einzugliedern. ${ }^{10}$

Diese beiden unmittelbaren Forderungen Napoleons erreichten den sächsischen König erst post festum, nachdem der Kaiser auch ohne jede militärische Mitwirkung der sächsischen Armee den Sieg bei Lützen über die verbündeten Russen und Preußen errungen hatte. Gerade dies aber erleichterte es dem sächsischen König, auf die im Serra-Text nicht ausdrücklich formulierte Frage, ob er sich noch als Mitglied des Rheinbundes sehe und daher willens sei, alle daraus erwachsenden Verpflichtungen zu übernehmen, ohne Umschweife bejahend $\mathrm{zu}$ antworten. Allerdings befand sich im Text des Dokuments noch eine kleine, aber hilfreiche Brücke: der Verweis darauf, dass es in der Umgebung des Königs übelwollende Personen gebe, namentlich den Minister der auswärtigen Angelegenheiten, den Grafen von Senfft.

Bekanntlich traf der König nach der Audienz, die er dem Baron von Serra gewährte, unverzüglich Maßnahmen, die zur Wiederherstellung der guten Beziehungen zum Kaiser von ihm erwartet wurden. Der Generalstabschef, Generalleutnant von Gersdorff, wurde mit einer Instruktion zur Öffnung der Festung Torgau für französische Truppen in Marsch gesetzt, analog hierzu hatte der General von Zeschau Befehle erhalten, die zur Öffnung der Festung Königstein für französische Truppen an den Kommandanten überbracht werden sollten.

10 In der Festung Torgau befanden sich zum fraglichen Zeitpunkt 11.000 sächsische Soldaten und 140 Offiziere unter dem Kommando des Generalleutnants von Thielmann. Auf Befehl des Königs wurde die Festung ab dem 20. April 1813 für alle fremden Truppen geschlossen. Dieser Befehl konnte nur im Einverständnis mit dem österreichischen Kaiser aufgehoben werden. 
Auf Befehl des Königs ${ }^{11}$ trat der Minister von Senfft noch am gleichen Tag, am 8. Mai 1813, von allen seinen Ämtern zurück, woraufhin der König den Chef des Militärdepartements, den General von Cerrini, mit der Wahrnehmung seiner bisherigen Geschäfte beauftragte. Friedrich August I. bereitete seine Rückkehr nach Dresden vor. Diese begann ab 10. Mai 1813. Zuvor unterrichtete er Kaiser Franz I., dass ab sofort alle getroffenen Abmachungen durch den Gang der Ereignisse (circonstances) aufgehoben seien. Lediglich der weitere Durchzug der Truppen des General von Gablenz und des Fürsten Poniatowski in Stärke von ca. 7.000 Mann über österreichisches Gebiet nach Sachsen war für ihn in diesem Moment noch von Interesse. ${ }^{12}$

Fragt man danach, ob der sächsische König unter diesen Umständen eine andere Entscheidung hätte treffen können, etwa zugunsten der Beibehaltung der Konvention vom 20. April 1813, so darf man nicht übersehen, was dabei für ihn auf dem Spiel stand. Napoleon hatte während des Vormarsches in Thüringen bei einem Gespräch mit dem Herzog von Sachsen-Weimar-Eisenach durchblicken lassen, dass er sich auch jemand anderen auf dem sächsischen Thron vorstellen könne. Herzog Karl August hatte diese Bemerkung ganz offensichtlich seinem königlichen Vetter nach Prag übermittelt. Der französische Gesandte in Weimar, St. Aignan, hatte seinerseits den Grafen von Senfft vom Inhalt dieses Gesprächs in Kenntnis gesetzt. Das bedeutet, dass mit dem Eintreffen der Nachricht mit dem tatsächlichen Ergebnis der Lützener Schlacht auch beim König und dessen Hofstaat in Prag Klarheit darüber entstand, dass es zum weiteren Verbleiben im Bündnis mit Frankreich keine wirkliche Alternative gab.

Die Begrüßung des Königs in Dresden am 12. Mai 1813 durch Napoleon war sehr feierlich und großmütig: Der Kaiser lobte seinen Verbündeten öffentlich vor den Magistratspersonen ausdrücklich für seine Bündnistreue. Wörtlich sagte er folgende wenige Sätze: Liebt Euren König: seht in ibm den Retter Sachsens! Wäre er seinem Worte weniger getreu, wäre er kein so redlicher Bundesgenosse gewesen, hätte er sich in den Meinungen Russlands und Preußens verstricken lassen, so wäre Sachsen verloren gewesen; ich würde es als erobertes Land behandelt haben. Meine Armee wird durch Sachsen nur durchmarschieren, und ibr werdet bald von den Beschwerden, die ibr bis jetzt zu ertragen habt, befreit werden. ${ }^{13}$ Napoleon wusste sehr genau, wovon er zu sprechen und worüber er zu schweigen hatte. Man darf sicher sein, dass der Kaiser seine Worte wohl erwogen hatte, um ungeachtet aller Absonderlichkeiten im Verhalten des sächsischen Königs über Wochen hinweg dieses Bündnis aufrechtzuerhalten.

\section{Anhang}

1. Nicht nummerierte Depesche des Ministers der ÄUsSeren Angelegenheiten GRAF SENFFT AN DEN BEVOLLMÄCHTIGTEN GESANDTEN DES SÄCHSISCHEN KÖNIGS AM WIENER HOF VON WATZDORF

Prag, 5. Mai 1813

Quelle: HStA Dresden, 10026 Geheimes Kabinett, Loc. 742/4, fol. 132v.

Senfft informiert besonnen Watzdorf über Lützen und fügt Dokumente bei; bittet um die Informierung des Grafen Metternich. Noch scheint alles offen zu sein.

11 Vgl. Schreiben des Grafen von Senfft vom 8. Mai 1813 aus Prag an den General von Watzdorf in Wien; vgl. HStA Dresden, 10026 Geheimes Kabinett, Loc. 742/4, fol. 149v.

12 HStA Dresden, 10026 Geheimes Kabinett, Loc. 2954, fol. 155v-r, Brief des Königs Friedrich August I. vom 10. Mai 1813 an den Kaiser Franz I. in Wien.

13 Dresdner Anzeigen für Jedermann, Nr. 106, 14. Mai 1813, Titelseite, Sp. 1. 
Monsieur,

Un Courier arrivé cet après midi de Dresde, nous a apporté des nouvelles authentiques sur le évènemens militaires qui ont en lieu aux environs de Leipzig du $1 \mathrm{r}$ jusqu'au 3 . du courant. Vous les trouverez, Monsieur, dans le rapport dont je joins une copie à cette Dépêche, et dont Vous Vous empresserez de faire part à S. E. Mr. le Comte de Metternich.

Je suis avec une considération très distinguée

Monsieur,

Votre très humble \& très

obéissant serviteur

Senfft

2. Kopie eines Schreibens des Barons de Serra, des französischen Gesandten am Dresdner Hof, an den KÖNIg Friedrich August I.; Beilage Zu einem Schreiben des Grafen von SenfFt an den sächsischen Gesandten in Wien, General von WATZDORF

Prag, 8. Mai 1813

Quelle: HStA Dresden, 10026 Geheimes Kabinett, Loc. 742/4, fol. 151v-152r.

Dieses entscheidende Schreiben ist eindeutig autorisiert durch Außerungen und Ermabnungen des Kaisers Napoleon an den sächsischen König, auf welche Weise er seit Jena und Tilsit den König als seinen Freund und Verbündeten begünstigt und bevorzugt habe. Deshalb sei es ihm völlig unbegreiflich, weshalb dieser in jüngster Zeit ihm wiederholt seine Bitte um Bereitstellung zweier Kavallerie-Regimenter abgeschlagen habe, wo er sie doch dringend auch zur Befreiung Sachsens benötigt habe.

Der Kaiser kannte König Friedrich August I. zu genau, um etwa nicht damit zu rechnen, dass dieser nach dem Sieg von Lützen in die Bündnisverpflichtungen gegenüber Frankreich zurückkehren würde. Das ,Banernopfer' aber hieß in diesem Falle Graf Senfft von Pilsach.

Ziemlich direkt wird dem König die Brücke gebaut, sich durch eine offene Deklaration zu den Verpflichtungen des Rheinbundes zu bekennen, womit die politische Zukunft eines Bündnisses mit Osterreich, wofür Senfft im Auftrag des Königs so zäh gearbeitet hatte, erledigt wäre. Schmeichelei und Bedrohung wechseln sich ständig ab. Der König konnte nicht absagen, wenn er Schlimmeres für seine Existenz vermeiden wollte. Die vagen Avancen des Kaisers hatten ibr Ziel, Sachsen in Reib und Glied zu stellen, durchaus erreicht. Der Zeitpunkt, nämlich der 8. Mai 1813, als die Nachricht vom Sieg des Kaisers bei Lützen bekannt geworden war, konnte nicht besser gewählt werden, König Friedrich August I. zur aktiven Rückkehr in das Bündnis zu veranlassen.

Das in älterer Geschichtsliteratur zuweilen als, Ultimatum' bezeichnete Schreiben sollte eher als ,Note', wie sie im diplomatischen Verkehr üblich war, angesehen werden.

[fol. 151v] S. M. l'Empereur \& Roi après avoir pris en sérieuse considération les motifs que S. M. le Roi de Saxe a voulu Lui exposer à l'appui du refus de mettre à sa disposition pour la cause commune les deux régimens de Cavalerie (Cuirassiers de la Garde \& de Zastrow) après avoir porté une grande attention à la réponse de $\mathrm{S}$. M. Royale dont Mr. le Gal de. Flahaut a été porteur, a commandé au Soussigné de faire à S. E. le Cte. de Senfft. Mintre. des Relations extérieures de S. M. le Roi de Saxe, Duc de Varsovie, les communications que les circonstances ont coigé \& coigerant. 
Il ne pourvoit être question d'appeler l'attention que S. M. Royale vouloir bien lui prêter, sur la justice \& la convenance de ces demandes. Ce sont des attributs que leur adjugeait le principe d'une cause commune, des engagements formels et la déclaration solennelle de S. M. l'Empereur, de ne pas abandonner ses Alliés, déclaration qu'il maintient par le fait en combattant en ce moment à la tête de Ses Armées pour la délivrance de la Saxe.

Le Soussigné n'a su adresser au cœur du Roi que le langage du cœur. Pourvoit il traiter autrement un sujet aussi auguste et aussi touchant par celui de amitié des deux Souverains, cette amitié qui a consacré l'union de leurs volontés, de leurs dispositions, de leurs intérêts? Cette amitié avoir été active, et agissante de part $\&$ d'autre jusqu'à ce jour. Pourquoi devoir elle cesser de l'être de la part du Roi, lorsqu'elle ne le fut jamais plus \& ne saurit l'être d'avantage qu'elle ne l'est actuellement de la part de l'Empereur.

Un changement quelconque du Rois que ses refus réitérés établissent [fol. 151r] quand même son cœur l désavouerait, pourrit il être fondé à l'égard de l'Empereur lui qui estima le Roi avant que des rapports plus positifs assent existé entre eux; lui qui vit à regret ce Prince entrainée il y a 7 ans à se joindre à ses ennemis, qui de Jena $\&$ de Posen lui tendit une main fraternelle qui créant à Tilsit deux nouveaux Etats en Europe en remit un au Roi, qui a maintenu l'inviolabilité de cet Etat dans toutes les négociations suivantes, qui la agrandi de plus beau fruit de ses triomphes dans la guerre de 1809, \& qui en soutient une nouvelle principalement pour défendre l'existence et les droits de ce même Duché de Varsovie? L'Empereur peut il n'être plus aux yeux du Roi ce bon Allié qui marchant à cette nouvelle guerre a voulu être d'abord son hôte pour être ensuite son défenseur, et qui s'il avoient de Moscow à Paris avec une rapidité qui sera l'étonnement de nos neveux s'arrête qu'à Dresde, pour vois ballé qui lui est cher, \& s'il retourne à Ses Armées entretient les Princes de la Confédération du Rhin réunis sur son passage à Mayence de la constance éprouvée du Roi de Saxe, de Sa fidélité dans Ses engagements et de la sincérité de son amitié envers lui?

Qu'est ce qui a pu arrêter avec une marche aussi noble et aussi digne d'un Prince renommé par Ses vertus et Sa sagesse? Qu'est a qui suspend tant à coup sa confiance si bien appréciée par son Auguste Allié? Cette confiance que le mal causé par le déchainement des Eléments à la fin de la dernière campagne n'avoir point ébranlée? Est ce la mauvaise volonté de quelques ans de ses sujets séduits par les ennemis communs ou le bruit d'un mauvaise esprit soumette parmi des troupes qui infidèles à leur pays poudroient joindre à l'amour d'un honteux repos, le crime de la désobéissance envers leur Souverain? Mais pour dissipes ces obstacles appréhendés il eut suffi au Roi de désapprouver hautement cet également, de commander la soumission à ses Ordres, mander \& rendre les Chefs militaires responsables de la résistance coupable des corps à ses volonté.

Est ce le désir ou l'agilité citée si souvent de conserver ces Corps pour l'avenir et pour une plus grande occasion? Mais lorsque les ennemis soulent les Etats du Roi, l'avenir peut il entrer en balance avec le présent, \& peut il y avoie une plus grande occasion, une occasion, plus propre d'employer ces moyens que celle que le meilleur juge de l'emploi de ces forces [fol. 152v] militaires a témoigné être la plus convenable, et peut être la seule ou allés puissent être d'une agilité réelle?

La France et les Co Etats de la Confédération doivent ils tout faire en cette campagne pour cause commune, pour la cause de la Saxe, et la Saxe doit elle être seule dissonée de faire ce qui est en con pouvoir d'y contribues! Cette Puissance ne vendrait elle agir désormais que lorsqu'on ne pourrit plus lui tenir compte d'une disposition à dessin retardée?

Sont ce des engagements nouvellement contractés qui paralyseraient les effets de l'amitié que le Roi proteste de conserver toute entière envers Son premier Allié?

Â la vérité des engagements soit ébauchés soit contractés dont ou avoir fait une misère à l'Empereur et Roi répondraient mal à la confiance que le Roi avoir mise sans réserve en lui, et cependant avoués de bonne foi ils poudroient se borner sans un plus grand inconvénient à partager les désirs et sollicitées d'une paix prochaine. 
Mais ces désires de la paix le besoin même de cette paix après bien des sacrifices, peuvent ils suspendre le concours obligatoire d'une des parties intéressés à poursuivre jusques là avec la même intention la guerre entreprise, le seul moyen efficace de parvenir au terme désiré?

Cas l'idée de la loyauté \& de la probité du Roi interdit encore à l'imagination la moins retenue de supposer que ces nouveaux engagements ont dérogé à ceux contractés précédemment par la Saxe et à ces obligations comme membre de la Confédération du Rhin, et comme Allié de la France dans la guerre actuelle?

Serait ce enfin l'incertitude ou la crainte des évènements militaires qui suspendrait en ce moment la bonne volonté du Roi envers Son Allié?

Qu'on suppose ces évènements du jour se déclares à l'avantage ou contre la France.

Dans le premier cas le Roi vendrait il assimile sa conduite à celle du vulgaire que la fortune détermine, et ne trouverait il pas bien plus conforme à Son grand caractère de se préserver par des dispositions anciennes du danger d'être en suite confondu dans l'opinion publique parmi ceux qui moins bien intentionnés que lui applaudiront ensemble au vainqueur?

Dans le second cas qui est ce qu'il y avoir de plus digne de la grandeur d'âme de Fréderic Auguste de dire à Son auguste Allié, j’offre de bon gré lorsque les circonstances peuvent ajouter quelque utilité à mon offre ce moyens qui me restent et que je refouloir tant que je pouvoir penser qu'inutiles dans le moment ils pourvoient être réservés [fol. 152r] sagement pour un besoin avenir.

Le Soussigné ayant ainsi relancé ce qu'il a en l'honneur de dire hier à S. M. Royale s'aperçoit d'avoir présenté à S. E. le Minre. des Relats. extrais de Saxe tout les motifs qui peuvent justement et pertinemment influer sur la détermination de sa Cour relativement aux demandes de S. M. I. \& R.

Ces demandes sont

1. que les deux régimens de Cuirassiers soyas mis à la disposition de S. M. l'Empereur pour l'intérêt \& le service de la cause commune, de manière que recevant les ordres de cétènes prêts, ils puissent être dirigés ensuite sur le point convenable que S. M. I. \& R. indiquera. S. M. l'Empereur avoir demandé cette disposition à S. M. le Roi comme une chose agréable qu'il lui serait, comme un service qui en devient tous les jours moins au. Il attache aujourd'hui au grand prix à cet acte de complaisance de la part de Son auguste Allié et ami, et le souvenir de cette complaisance ne saurit être limité par le temps.

2. que S. M. Royale vermille donner des ordres au Général Commandant de Torgau, pourque le Gal. Reynier du moment que cette place sera débloqué puisse prendre le commandement du 7e Corps ou contingent Saxon qui s'y est rassemblé. S. M. l'Empereur \& Roi fort de Son génie de sa puissance et du dévouement sans bornes des sujets pour sa personne et pour sa gloire peut seul ne pas compter le nombre de ses Ennemis. Il peut avoies encore un ami de moins. Mais Son cœur suffira de ce que le refus de ces demandes ne lui laissera plus de doute que S. M. le Roi est cet ami qu'il aurait encore perdu. Ce regret que S. M. le Roi partagerait sans doute pourrit il être infructueuse, et sera t-il tout ce qui récitera d'une si belle amitié a deux Souverains dont elle faisait le bonheur?

Le Soussigné on se flatter que les Démarches qu'il a en l'honneur de faire pourront engager S. M. Royale à écarter ce malheur, et il se réserve s'il étroit réduit à ce convaincre du contraire de faire une déclaration ultérieure.

Il a l'honneur d'offris \& $\mathrm{c}$.

pp.

(signé) Baron de Serra. 


\section{BRief Des Kaisers NAPOleon AN DEN BARON VON SERRA}

Mainz, 20. April 1813

Quelle: Lettres inédites de Napoleon Ier, Publiées par Léon Lecestre, Tome second (18101815), Paris Libration Plon 1897, S. 232 f.

\section{[S. 232] Au Baron de Serra}

Ministre plénipotentaire près de Roi de Saxe ${ }^{14}$

Je ne puis vous témoigner mon extrême mécontentement de ce que vous avez renvoyé mon officier d'ordonnance Lauriston sans réponse. Vous savez que le Roi de Saxe a 1800 hommes de cavalerie, dont j'ai tout besoin, et vous ne dites rien! Votre inertie est inconvenable. Demander catégoriquement si on veut faire parti cette cavalerie, et, dans ce cas, qu'on la dirigé soit sur Wurzbourg, soit sur le général Bertrand, qui est à Cobourg. Vous devez faire connaître que je considérais au refus, comme un commencement de changement de système, Je n'avais pas a m'attendre à cela du roi après ce que j'ai fait pour cette cour. Quant à vous, il est inconvenable, que vous ayez poussé l'oubli des convenances au point de laisser partir le baron Lauriston sans réponse. Ne gardez ce courrier que douze heures, envoyez-moi par son retour un état de situation de cette cavalerie. Le général Bertrand n'est qui à Cobourg cette cavalerie lui sera très utile.

Je vous envoie la lettre que j'écris au roi. ${ }^{15} \mathrm{Si}$ dans l'intervalle qui est écoulé, le roi avait, fait droit à ma demande et fait partir en cavalerie, vous ne lui remettrez pas ma lettre. Si, au cas contraire, il n'avait encore rien fait, vous cachetterez cette lettre et la lui remettriez, ignorant ce que qu'elle contient.

Je suppose aussi que le roi aura donné les ordres au général Thielmann à Torgau, pour que aussitôt que les communications seront rétablies le général Reynier puisse répondre le commandement des troupes, et que je puisse en disposer, ainsi que de la garnison, selon que je le jugerai nécessaire.

Vous avez dû savoir le 17 mon départ de Paris, je suis surpris que vous ne m'avez pas sur-lechamp expédié un courrier sur Mayence, pour m'instruire de tout ce qu'il y a de nouveau. Si ce courrier était parti le 18, je devrais le recevoir dans la journée.

Ecrivez à M. de Mercy que le prince de Moskowa est arrivée à Weimar, que mon quartier général se portée sur Erfurt, et que tout est en mouvement: que je désire qu'il fasse des instances [S. 233] que le général de Wrede prenne le commandement des troupes bavaroises, s'il est en état, et que les instructions, à donner aux commandants soient telles, qu'on obéisse à mes ordres sans restrictions.

Je désire que vous m'expédiiez un courrier tous les jours, pour m'instruire de tout ce qui arrivera votre connaissance par les Saxons, soit par les Bavarois. Vos courriers passeront par Würtzbourg et s'informèrent auprès de mon ministre où je suis.

P.-S. - En pensant mieux, je préfère expédier, mon aide de camp Flahaut, qui sera porteur de ma lettre au roi et de la présente lettre pour vous.

14 AF IV 809.

15 Correspondance nr. 19886. 